\title{
Thermographic survey of frescoes with different thermal stimuli: a PLS-based analysis
}

\author{
by P. Bison*, A. Bortolin*, G. Cadelano*, G. Ferrarini**, L. Finesso***, K. Mouhoubi****, J.L Bodnar**** \\ *ITC-CNR, Corso Stati Uniti 4, 35127 Padova Italy, paolo.bison@itc.cnr.it \\ **DII-UNIPD, Via Venezia 1, 35131, Padova, Italy \\ ***IEIIT-CNR, Via Gradenigo 6/B, 35131, Padova, Italy \\ ${ }^{* * * * G R E S P I / C A T H E R M, ~ U F R ~ S c i e n c e s ~ E x a c t e s ~ e t ~ N a t u r e l l e s, ~ B P ~ 1039, ~} 51687$ Reims cedex 02
}

\begin{abstract}
Infrared thermography is a well-known technique for the non-destructive evaluation of artworks. In recent times, several image processing algorithms have been proposed and studied to enhance the detection of defects. Amongst them, post-processing methods based on Partial Least Squares (PLS) have shown good results in terms of reliability and detectability of defects. This work analyzes the available PLS-related algorithms for the evaluation of a fresco sample with known defects, stimulated with different heating sources. The results are compared in terms of Signal to Noise Ratio and Receiver Operating Characteristic curves.
\end{abstract}

\section{Introduction}

The advantages related to the application of Infrared Thermography (IRT) in the field of non-destructive testing and evaluation have been presented to the scientific community since long time [1]. IRT is widely utilized for the analysis of different materials and components, and its non-invasive and contactless nature promotes it as a suitable technique for the inspection of artworks [2]. Cultural heritage is typically surveyed to find hidden structure or information, to determine the presence of moisture related damage, to locate delamination or detachments [3]. The standard inspection procedure consists in imposing a heat flux on the object and measure the related temperature variation with a thermal camera. The obtained thermal images results are successively processed with dedicated algorithms. Several options are available in the literature [4] and in recent times PLS-based methods have shown good results both for industrial applications [5] and for cultural heritage, especially for frescoes [6].

\section{Materials and methods}

The analyzed fresco specimen is a plaster panel sizing $68 \times 50 \times 5 \mathrm{~cm}$. The panel surface has been divided in twenty sectors, painted with different colors. Under the surface 32 defects have been created, inserting circular inclusions of polystyrene of diameter $1.5 \mathrm{~cm}$ and thickness equal to $1 \mathrm{~mm}$. Two different testing procedures have been applied to the specimen. In the first one a traditional pulsed heating, delivered by lamps, has been applied. In the second one, a Pseudo Random Binary Signal (PRBS) was sent to the lamps that generated a series of short heating steps turning on and off the lamps. This technique minimizes the temperature variation of the specimen, that is a primary necessity when dealing with cultural heritage. In both cases a thermal camera (FLIR 655 SC, spectral range from 7.5 to $14 \mu \mathrm{m}$, declared thermal sensitivity of $30 \mathrm{mK}$ ) recorded a sequence of thermal images at $1 \mathrm{hz}$, for $4-8$ minutes depending on the heating source.

The acquired images have been analyzed with PLS-based techniques. PLS is a statistical method that integrates Principal Component Analysis and Multiple Linear Regression. The basic equations of PLS are

$$
\begin{aligned}
& X=T P^{T}+E \\
& Y=T Q^{T}+F
\end{aligned}
$$

The data matrix could be the $X$ or the $Y$ matrix, leading in the first case to the PLST approach and in the second case to the PLSRT approach [6]. 


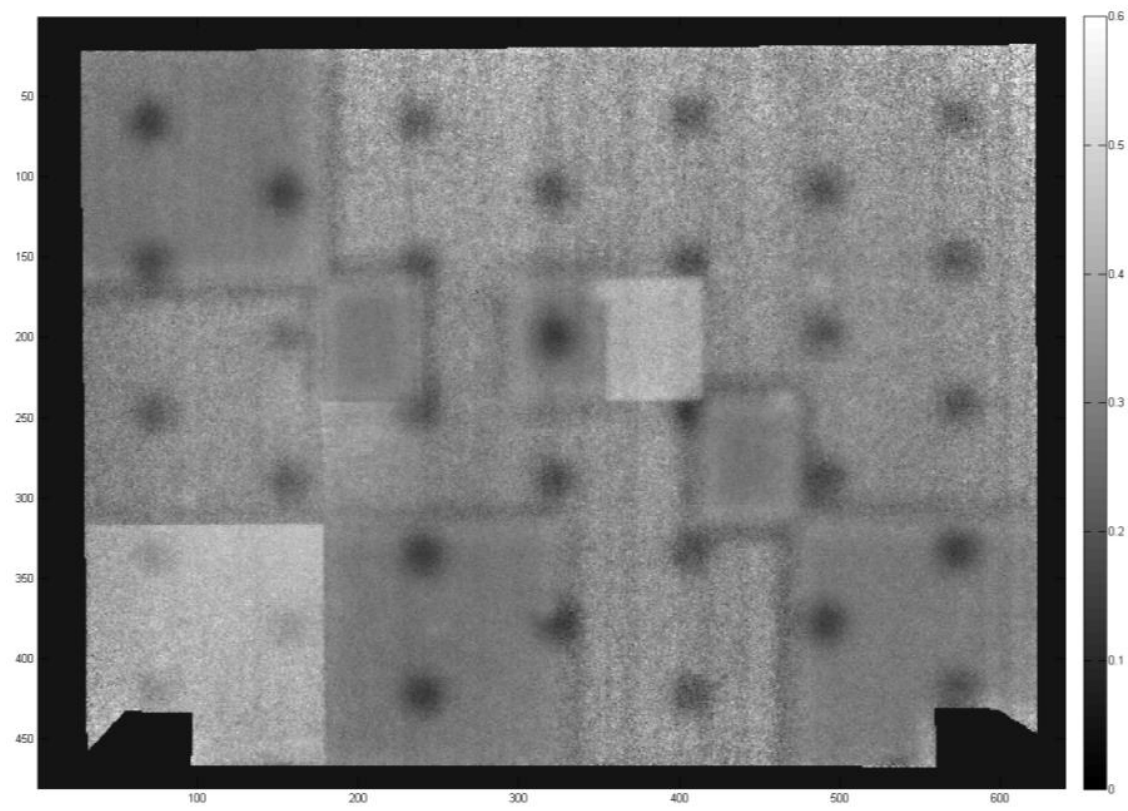

Fig. 1. Result of the Partial Least Square Thermography algorithm applied to the fresco specimen stimulated with a pulsed heating.

As the defect map is known, the results obtained on the specimen under two different heating conditions and with the different PLS-based post-processing techniques have been compared on the Signal to Noise Ratio, to determine the effectiveness of the method, and with the Receiver Operating Characteristic curves, that give a measure of the reliability of the method quantifying its sensitivity.

\section{REFERENCES}

[1] Maldague X., "Theory and Practice of infrared technology for nondestructive testing", Wiley, 2001.

[2] Cadelano G., Bison P., Bortolin A., Ferrarini G., Girotto M., Peron F., Volinia M., "Conservation of historical frescoes by timed infrared imaging analysis", AITA 2013 - Advanced Infrared Technology and Applications, Torino (Italy), 2013.

[3] Carlomagno, G.M., Meola, C.,"Comparison between thermographic techniques for frescoes NDT“, NDT and E International, Volume 35, Issue 8, December 2002, Pages 559-565.

[4] Bendada A., Sfarra S., Ambrosini D., Paoletti D., Ibarra-Castanedo C., Maldague X., "Active thermography data processing for the NDT\&E of frescoes", 10th International Conference on Quantitative InfraRed Thermography July 27-30, 2010, Québec (Canada)

[5] Lopez F., Nicolau V., Maldague X., Ibarra-Castanedo C., "Multivariate infrared signal processing by partial least squares thermography", Proceedings of the $16^{\text {th }}$ International Symposium on Applied Electromagnetics and Mechanics, Québec (Canada), 2013.

[6] P. Bison, A. Bortolin, G. Cadelano, G. Ferrarini, L. Finesso, F. Lopez,X. Maldague, "Evaluation of frescoes detachments by partial least square", Proceedings of QIRT 2014, Bordeaux. 